\title{
The association between intake of whole grain, refined grain, fast food and carbonated drinks with depression and quality of life in Iranian adolescent girls
}

\author{
Abbas Ali Sangouni \\ Shahid Sadoughi University of Medical Sciences and Health Services \\ zahra darabi \\ Shahid Sadoughi University of Medical Sciences and Health Services \\ Majid Ghayour-Mobarhan \\ Mashhad University of Medical Sciences \\ Mohsen Mazidi \\ King's College London \\ Katie E Lane \\ Liverpool John Moores University \\ Sayyed Saeid Khayyatzadeh ( Khayyatzadehss@yahoo.com ) \\ Shahid Sadoughi University of Medical Sciences and Health Services
}

Research Article

Keywords:

Posted Date: February 24th, 2022

DOI: https://doi.org/10.21203/rs.3.rs-1249126/v2

License: (c) (1) This work is licensed under a Creative Commons Attribution 4.0 International License. Read Full License 


\section{Abstract}

Background:

We evaluated the association between fast food, whole grain, refined grain and carbonated drink consumption with depression and QoL in Iranian adolescent girls.

Methods:

In this cross-sectional study, 733 adolescent girls were included from Mashhad and Sabzevar cities in northeastern Iran. The Beck Depression Inventory (BDI) and SF-12v2 questionnaire were used to assess depression and QoL, respectively. A valid and reliable food frequency questionnaire was used to obtained dietary intakes of the study participants. To explore the association between intake of food groups and depression and QoL, we used linear and logistic regression in crude and adjusted models.

Results:

There was a significant decreasing trend in the odds of poor QOL and depression across increasing quartile intake of whole grain; but, there was no linear relationship between whole grain intake with depression and QoL score. In addition, subjects in the highest quartile of carbonated drink consumption had higher odds of poor QoL compared to the first quartile (OR: 1.33; $95 \% \mathrm{Cl}: 1.00-2.32, \mathrm{P}=0.04$ ). We found a positive association between intake of fast food and depression score $(\beta=0.09)$. Intake of fast food was inversely associated with $Q$ oL score $(\beta=-0.081)$. However, there was no significant relationship between intake of refined grains and carbonated drinks with depression, and between whole grain and refined grain intake with QoL.

Conclusions:

We demonstrated an inverse association between fast food intake with depression and QOL. To better conclude, further studies evaluating the association of various food groups with depression and QoL.

\section{Introduction}

Recent evidence confirms the growing prevalence as well as socioeconomic costs of mental health disorders (1-3). Depression is a common mental health disorder, with two major symptoms including depressed mood and anhedonia, which are often accompanied with several other symptoms (3). The lifetime prevalence of depression is estimated to be $15 \%$ in the general population (4). Many patients suffering from depression experience depressive symptoms during adolescence (5). The prevalence of depression is affected by gender and is higher among adolescent females than males $(6,7)$. Obesity, oxidative stress, and inflammation are linked to the development and progression of depressive symptoms (8-10). Based on a meta-analysis the prevalence of depression among Iranian children and adolescents is reported to be $43.5 \%(11)$.

Several studies have suggested that depression has a negative effect on quality of life (QoL) $(12,13)$. QoL is a multidimensional concept (14). Based on the World Health Organization (WHO), QoL is defined as follows: "the perception of individuals about their position in life in the context of the culture and value systems which is related to their goals, expectations, standards and concerns" (15).

Previous studies have proposed that diet can play an important role in the development or preventive of depression (16-18). Several studies have investigated the association between dietary patterns, intake of nutrients or specific food groups with depression (16-20). Results of some studies reported that intake of copper, iron, zinc and selenium were inversely associated with depressive symptoms $(19,20)$. Results of other studies showed that healthy dietary patterns which provide higher amounts of gruel, oatmeal, whole grains, fresh yellow or red vegetables, fruits, soya milk and fish, can decrease risk of depression among adolescents $(16,17)$. Unhealthy dietary patterns like the western dietary pattern that are characterized by high intake of red and processed meats, refined foods, saturated fatty acids (SFAs), noodles, white bread and coffee can increase the risk of depression $(16,18)$. Investigations suggested that intake of specific food groups such as vegetables, fruits, nuts and legumes have an inverse association with the risk of depression (21, 22). There are few studies investigating the relationship between specific food groups with depression. A recent study has evaluated the relationship between diet and QoL (23); however, there no published investigations evaluating the association between specific food groups and QoL. Accordingly, the present study aimed to investigate the association between intake of refined grains, whole grains, fast foods and carbonated drinks with depression and QoL in adolescent girls.

\section{Methods And Materials}

\section{Study population}

This cross sectional study was conducted among 733 adolescent girls (aged 12-18 years) in January 2015. Participants were selected from several schools in the cities of Mashhad and Sabzevar, in northeastern Iran by a random cluster sampling method. Adolescents with history of autoimmune diseases, cancer, metabolic bone disease, hepatic or renal failure, cardiovascular disorders, malabsorption or thyroid, parathyroid, adrenal diseases and anorexia nervosa or bulimia were excluded from the study. In addition, participants who were included, did not receive anti-inflammatory, anti-depressant, antidiabetic, or antiobesity drugs, vitamin D or calcium supplement, and any hormone therapy within the last 6 months. Before the start of the study, written informed consent forms confirmed by the ethical committee of Mashhad University of Medical Sciences, in Mashhad, Iran (ethical code number: 931188 ) were completed by all participants and their parents.

Demographic and anthropometric assessments 
Demographic variables were assessed using a demographic questionnaire administered by trained interviewers. Demographic variables were age, smoking status, menstruation status, medical history, supplement intake, taking psychological treatment and chronic diseases. Participants' physical activity was assessed using a modifiable activity questionnaire (MAQ) (20). Physical activity level was calculated based on metabolic equivalent task minutes per week. Anthropometric variables such as weight, height and waist circumference (WC) were measured by the trained investigators using the standard protocols. Body mass index (BMI) computed as weight $(\mathrm{kg})$ divided by square of height $\left(\mathrm{m}^{2}\right)$.

Dietary assessment

Dietary intakes were estimated by a validated food-frequency questionnaire (FFQ) with 147 food items. The validity and reliability of this FFQ were confirmed previously (24). To complete the FFQ, a face-to-face interview was performed by a trained dietitian. To evaluate the frequency of food items intake during the last year, the participants answered the questions about their daily, weekly, monthly and yearly intake. The reported portion sizes in the FFQ were converted to grams using household measures, and the energy and nutrient intakes were calculated using the Nutritionist IV software (25).

Assessment of depression

Depression was assessed via a Persian version of the Beck Depression Inventory (BDI). This questionnaire has 21 items evaluating various symptoms of depression including feelings of guilt, feelings of hopelessness, sadness, crying, sleep disturbance, fear and loss of appetite over the past 2 weeks. The range of scores for the BDI was between 0 and 63 points. scores $0-13,14-19,20-28$ and 29-63 refer to no depression, mild depression, moderate depression, and severe depression respectively. The validity and reliability of this questionnaire were confirmed by previous studies $(26,27)$.

Quality of life assessment

To assess health-related QOL, the SF-12v2 questionnaire was used. This questionnaire is a short form of the SF-36 questionnaire and improved version of SF$12 \mathrm{v} 1$ (28). The validity and reliability of this questionnaire were approved in Iran (29). The questionnaire has 12 items which evaluate 8 domains of health including physical functioning, role limitations because of physical problems, bodily pain, general health, vitality, social functioning, role limitations because of emotional problems, and mental health. The range of QoL scores are between 0 (the worst QoL) to 100 (the best QoL). The median of the QoL score is 43 . The subjects were categorized as high QoL if their scores were higher than 43.

\section{Statistical analysis}

Subjects were classified into four groups across quartiles of their food group scores including whole grain, refined grain, carbonated drink and fast foods. General characteristics and nutrient intake across quartiles of food group score were expressed as means \pm SDs for continuous variables, and as numbers and percentages for categorical variables. To examine the differences between quartiles, one-way-ANOVA and Chi square test were used for continuous and categorical variable respectively. To investigate the relationship between quartiles of food group scores with depression and poor QoL, multivariate regression was conducted in the crude and adjusted models. Multivariate linear regression was used to explore the association between QOL score and depression with fast food, carbonated drink, whole grain and refined grain intake. Age and energy intake were adjusted in Model I. Additionally, BMI percentile was adjusted in Model II. Finally, physical activity, age, energy intake and BMI percentile were adjusted in the model III. All statistical analyses were conducted using the SPSS version 21. P-values less than 0.05 were considered as significant.

\section{Results}

General characteristics study participants

The mean age of the participants was 14.5 years. The prevalence of depression and poor QoL were $24 \%$ and $49 \%$, respectively. General characteristics and anthropometric indices of the participants across quartiles of food groups are demonstrated in Table 1. Participants in the lowest quartile of whole grain and the highest quartile of refined grain were significantly younger $(p=0.02$ and $p=0.01$, respectively). Anthropometric variables and other general characteristics were not different among quartiles of food intake. 
Table 1

General characteristics and Anthropometric indices of study participants by quartiles of food groups.

\begin{tabular}{|c|c|c|c|c|c|c|c|c|c|c|c|c|}
\hline & \multicolumn{3}{|c|}{ Whole grain } & \multicolumn{3}{|c|}{ Refined grain } & \multicolumn{3}{|c|}{ Fast food } & \multicolumn{3}{|c|}{ Carbonated drink } \\
\hline & Q1 & Q4 & $\begin{array}{l}P \\
\text { value }^{1}\end{array}$ & Q1 & Q4 & $\begin{array}{l}P \\
\text { value }^{1}\end{array}$ & Q1 & Q4 & $\begin{array}{l}\mathrm{P} \\
\text { value }^{1}\end{array}$ & Q1 & Q4 & $\begin{array}{l}\mathrm{P} \\
\text { value }^{1}\end{array}$ \\
\hline Age(year) & $\begin{array}{l}14.28 \pm \\
1.43\end{array}$ & $\begin{array}{l}14.75 \pm \\
1.62\end{array}$ & 0.02 & $\begin{array}{l}14.62 \pm \\
1.60\end{array}$ & $\begin{array}{l}14.14 \pm \\
1.36\end{array}$ & 0.01 & $\begin{array}{l}14.42 \pm \\
1.46\end{array}$ & $\begin{array}{l}14.60 \pm \\
1.57\end{array}$ & 1.00 & $\begin{array}{l}14.25 \pm \\
1.52\end{array}$ & $\begin{array}{l}14.66 \pm \\
1.58\end{array}$ & 0.07 \\
\hline $\mathrm{BMI}(\mathrm{Kg} / \mathrm{m} 2)$ & $\begin{array}{l}20.81 \pm \\
4.14\end{array}$ & $\begin{array}{l}21.59 \pm \\
4.12\end{array}$ & 0.46 & $\begin{array}{l}21.61 \pm \\
4.15\end{array}$ & $\begin{array}{l}20.91 \pm \\
3.93\end{array}$ & 0.68 & $\begin{array}{l}20.89 \pm \\
4.83\end{array}$ & $\begin{array}{l}21.46 \pm \\
4.05\end{array}$ & 1.00 & $\begin{array}{l}21.14 \pm \\
5.06\end{array}$ & $\begin{array}{l}21.27 \pm \\
4.15\end{array}$ & 1.00 \\
\hline Weight(Kg) & $\begin{array}{l}51.17 \pm \\
11.11\end{array}$ & $\begin{array}{l}54.11 \pm \\
11.62\end{array}$ & 0.11 & $\begin{array}{l}53.86 \pm \\
11.84\end{array}$ & $\begin{array}{l}52.31 \pm \\
11.05\end{array}$ & 1.00 & $\begin{array}{l}52.01 \pm \\
13.91\end{array}$ & $\begin{array}{l}53.54 \pm \\
11.06\end{array}$ & 1.00 & $\begin{array}{l}52.52 \pm \\
14.11\end{array}$ & $\begin{array}{l}53.14 \pm \\
11.80\end{array}$ & 1.00 \\
\hline $\begin{array}{l}\text { Waist circumference } \\
(\mathrm{cm})\end{array}$ & $\begin{array}{l}70.01 \pm \\
9.57\end{array}$ & $\begin{array}{l}70.53 \pm \\
8.62\end{array}$ & 1.00 & $\begin{array}{l}71.41 \pm \\
9.23\end{array}$ & $\begin{array}{l}70.15 \pm \\
8.45\end{array}$ & 1.00 & $\begin{array}{l}69.99 \pm \\
9.87\end{array}$ & $\begin{array}{l}70.29 \pm \\
8.70\end{array}$ & 1.00 & $\begin{array}{l}70.65 \pm \\
10.61\end{array}$ & $\begin{array}{l}70.60 \pm \\
9.13\end{array}$ & 1.00 \\
\hline $\begin{array}{l}\text { Metabolic equivalent } \\
\text { task(h/week) }\end{array}$ & $\begin{array}{l}44.74 \pm \\
3.39\end{array}$ & $\begin{array}{l}46.01 \pm \\
3.60\end{array}$ & $0.01>$ & $\begin{array}{l}45.27 \pm \\
2.95\end{array}$ & $\begin{array}{l}45.07 \pm \\
3.80\end{array}$ & 1.00 & $\begin{array}{l}45.00 \pm \\
2.71\end{array}$ & $\begin{array}{l}45.49 \pm \\
3.62\end{array}$ & 1.00 & $\begin{array}{l}44.84 \pm \\
2.89\end{array}$ & $\begin{array}{l}45.68 \pm \\
3.92\end{array}$ & 0.12 \\
\hline Score of depression & $\begin{array}{l}12.23 \pm \\
10.38\end{array}$ & $\begin{array}{l}10.23 \pm \\
8.66\end{array}$ & 0.24 & $\begin{array}{l}11.10 \pm \\
9.18\end{array}$ & $\begin{array}{l}11.25 \pm \\
9.42\end{array}$ & 1.00 & $\begin{array}{l}10.12 \pm \\
9.3\end{array}$ & $\begin{array}{l}11.27 \pm \\
8.65\end{array}$ & 1.00 & $\begin{array}{l}10.60 \pm \\
9.62\end{array}$ & $\begin{array}{l}11.48 \pm \\
9.80\end{array}$ & 1.00 \\
\hline Score of quality of life & $\begin{array}{l}41.34 \pm \\
7.69\end{array}$ & $\begin{array}{l}42.27 \pm \\
7.69\end{array}$ & 0.81 & $\begin{array}{l}41.95 \pm \\
7.72\end{array}$ & $\begin{array}{l}41.27 \pm \\
1.69\end{array}$ & 1.00 & $\begin{array}{l}42.07 \pm \\
8.64\end{array}$ & $\begin{array}{l}41.89 \pm \\
7.89\end{array}$ & 1.00 & $\begin{array}{l}42.50 \pm \\
8.34\end{array}$ & $\begin{array}{l}40.75 \pm \\
7.67\end{array}$ & 0.23 \\
\hline \multicolumn{13}{|l|}{ Values are means \pm SD } \\
\hline
\end{tabular}

Dietary intake of study participants

Dietary intake of study participants across quartiles of food groups are shown in Table 2. Participants in the highest quartiles of food groups (whole grain, refined grain, fast food and carbonated drink) compared with the participants who were in the lowest quartile had higher intake of energy, protein and carbohydrate. Vitamin B6 intake was significantly higher among participants in the last quartile of fast food intake (per $1000 \mathrm{kcal}$ ) compared to the first quartile $(p=0.04)$. Intake of Vitamin B12 was not significantly different between quartiles of food groups (per $1000 \mathrm{kcal}$ ). Participants in the first quartile of carbonated drink as well as fast foods intake had higher intake of folate compare with the fourth quartile (per $1000 \mathrm{kcal})(\mathrm{p}<0.001)$. The participants with a greater intake of refined grain had a lower intake of vitamin A ( $p=0.01)$, vitamin $C(p<0.001)$, and calcium (per $1000 \mathrm{kcal})(\mathrm{p}<0.001)$. Participants in the highest quartile of food groups intake including fast foods, refined grain and carbonated drink had higher intake of monounsaturated fatty acids (MUFA), polyunsaturated fatty acids (PUFAs) and SFAs. 
Table 2

Macronutrient and some micronutrients intake of study participants by quartiles of food group

\begin{tabular}{|c|c|c|c|c|c|c|c|c|c|c|c|c|}
\hline & \multicolumn{3}{|c|}{ Whole grain } & \multicolumn{3}{|c|}{ Refined grain } & \multicolumn{3}{|l|}{ Fast food } & \multicolumn{3}{|c|}{ Carbonate drink } \\
\hline & Q1 & Q4 & $\begin{array}{l}\mathrm{P} \\
\text { value }^{1}\end{array}$ & Q1 & Q4 & $\begin{array}{l}P \\
\text { value }^{1}\end{array}$ & Q1 & Q4 & $\begin{array}{l}\mathrm{P} \\
\text { value }^{1}\end{array}$ & Q1 & Q4 & $\begin{array}{l}\mathrm{P} \\
\text { value }^{1}\end{array}$ \\
\hline Energy(kcal) & $\begin{array}{l}2564.82 \\
\pm 869.47\end{array}$ & $\begin{array}{l}2878.28 \\
\pm 862.30\end{array}$ & $0.01>$ & $\begin{array}{l}2192.65 \\
\pm 777.85\end{array}$ & $\begin{array}{l}3337.90 \\
\pm 654.97\end{array}$ & $0.001>$ & $\begin{array}{l}2287.98 \\
\pm 781.87\end{array}$ & $\begin{array}{l}3166.93 \\
\pm 695.43\end{array}$ & $0.001>$ & $\begin{array}{l}2487.00 \\
\pm 857.97\end{array}$ & $\begin{array}{l}3135.92 \\
\pm 797.07\end{array}$ & $0.001>$ \\
\hline Carbohydrate(gr) & $\begin{array}{l}349.82 \\
\pm 127.1\end{array}$ & $\begin{array}{l}406.27 \\
\pm 123.91\end{array}$ & $0.001>$ & $\begin{array}{l}279.24 \\
\pm 100.79\end{array}$ & $\begin{array}{l}488.93 \\
\pm 25.67\end{array}$ & $0.001>$ & $\begin{array}{l}314.41 \\
\pm 116.71\end{array}$ & $\begin{array}{l}430.19 \\
\pm 112.52\end{array}$ & $0.001>$ & $\begin{array}{l}341.11 \\
\pm 123.98\end{array}$ & $\begin{array}{l}433.11 \\
\pm 117.68\end{array}$ & $0.001>$ \\
\hline Protein(gr) & $\begin{array}{l}86.49 \pm \\
33.31\end{array}$ & $\begin{array}{l}101.01 \\
\pm 31.99\end{array}$ & $0.001>$ & $\begin{array}{l}74.55 \pm \\
29.83\end{array}$ & $\begin{array}{l}114.18 \\
\pm 25.67\end{array}$ & $0.001>$ & $\begin{array}{l}78.78 \pm \\
29.57\end{array}$ & $\begin{array}{l}107.21 \\
\pm 29.20\end{array}$ & $0.001>$ & $\begin{array}{l}88.43 \pm \\
32.35\end{array}$ & $\begin{array}{l}100.95 \\
\pm 31.52\end{array}$ & $0.01>$ \\
\hline Fat(gr) & $\begin{array}{l}97.18 \pm \\
41.63\end{array}$ & $\begin{array}{l}102.85 \\
\pm 40.81\end{array}$ & 1.00 & $\begin{array}{l}92.04 \pm \\
4.67\end{array}$ & $\begin{array}{l}112.17 \\
\pm 38.34\end{array}$ & $0.001>$ & $\begin{array}{l}85.42 \pm \\
40.33\end{array}$ & $\begin{array}{l}121.28 \\
\pm 35.7\end{array}$ & $0.001>$ & $\begin{array}{l}92.43 \pm \\
42.21\end{array}$ & $\begin{array}{l}119.58 \\
\pm 40.81\end{array}$ & $0.001>$ \\
\hline Cholesterol(mg) & $\begin{array}{l}229.57 \\
\pm 166.75\end{array}$ & $\begin{array}{l}240.62 \\
\pm 112.79\end{array}$ & 1.00 & $\begin{array}{l}225.75 \\
\pm 150.69\end{array}$ & $\begin{array}{l}251.55 \\
\pm 139.33\end{array}$ & 0.43 & $\begin{array}{l}209.24 \\
\pm 131.38\end{array}$ & $\begin{array}{l}269.32 \\
\pm 129.35\end{array}$ & $0.001>$ & $\begin{array}{l}237.71 \\
\pm 173.45\end{array}$ & $\begin{array}{l}245.81 \\
\pm 128.88\end{array}$ & 1.00 \\
\hline $\begin{array}{l}\text { Saturated fatty } \\
\text { acid (gr) }\end{array}$ & $\begin{array}{l}27.78 \pm \\
12.61\end{array}$ & $\begin{array}{l}30.88 \pm \\
13.00\end{array}$ & 0.16 & $\begin{array}{l}28.08 \pm \\
14.49\end{array}$ & $\begin{array}{l}32.26 \pm \\
11.57\end{array}$ & 0.01 & $\begin{array}{l}25.17 \pm \\
12.50\end{array}$ & $\begin{array}{l}36.81 \pm \\
13.05\end{array}$ & $0.001>$ & $\begin{array}{l}27.89 \pm \\
14.03\end{array}$ & $\begin{array}{l}34.07 \pm \\
12.63\end{array}$ & $0.001>$ \\
\hline $\begin{array}{l}\text { Monounsaturated } \\
\text { fatty acid(gr) }\end{array}$ & $\begin{array}{l}31.44 \pm \\
13.13\end{array}$ & $\begin{array}{l}32.78 \pm \\
13.72\end{array}$ & 1.00 & $\begin{array}{l}28.91 \pm \\
15.06\end{array}$ & $\begin{array}{l}36.45 \pm \\
14.00\end{array}$ & $0.001>$ & $\begin{array}{l}27.76 \pm \\
14.58\end{array}$ & $\begin{array}{l}38.90 \pm \\
12.06\end{array}$ & $0.001>$ & $\begin{array}{l}30.41 \pm \\
15.10\end{array}$ & $\begin{array}{l}38.07 \pm \\
14.17\end{array}$ & $0.001>$ \\
\hline $\begin{array}{l}\text { Polyunsaturated } \\
\text { fatty acid(gr) }\end{array}$ & $\begin{array}{l}22.55 \pm \\
11.22\end{array}$ & $\begin{array}{l}22.48 \pm \\
11.98\end{array}$ & 1.00 & $\begin{array}{l}19.91 \pm \\
12.91\end{array}$ & $\begin{array}{l}25.14 \pm \\
11.59\end{array}$ & $0.001>$ & $\begin{array}{l}19.08 \pm \\
11.91\end{array}$ & $\begin{array}{l}26.86 \pm \\
10.00\end{array}$ & $0.001>$ & $\begin{array}{l}20.86 \pm \\
11.38\end{array}$ & $\begin{array}{l}27.36 \pm \\
12.75\end{array}$ & $0.001>$ \\
\hline Fiber(gr) & $\begin{array}{l}43.04 \pm \\
23.19\end{array}$ & $\begin{array}{l}49.23 \pm \\
20.21\end{array}$ & 0.02 & $\begin{array}{l}33.51 \pm \\
14.80\end{array}$ & $\begin{array}{l}58.32 \pm \\
22.72\end{array}$ & $0.001>$ & $\begin{array}{l}45.01 \pm \\
22.17\end{array}$ & $\begin{array}{l}48.96 \pm \\
20.19\end{array}$ & $0.01>$ & $\begin{array}{l}42.59 \pm \\
19.8\end{array}$ & $\begin{array}{l}48.53 \pm \\
19.42\end{array}$ & 0.03 \\
\hline $\begin{array}{l}\text { Calcium(mg/1000 } \\
\text { Kcal) }\end{array}$ & $\begin{array}{l}411.61 \\
\pm 132.57\end{array}$ & $\begin{array}{l}422.84 \\
\pm 124.92\end{array}$ & 1.00 & $\begin{array}{l}459.20 \\
\pm 154.6\end{array}$ & $\begin{array}{l}382.14 \\
\pm 96.18\end{array}$ & $0.001>$ & $\begin{array}{l}433.94 \\
\pm 155.50\end{array}$ & $\begin{array}{l}410.97 \\
\pm 130.75\end{array}$ & 0.62 & $\begin{array}{l}436.94 \\
\pm 149.89\end{array}$ & $\begin{array}{l}395.71 \\
\pm 118.71\end{array}$ & 0.02 \\
\hline $\begin{array}{l}\text { Iron(mg/1000 } \\
\text { Kcal) }\end{array}$ & $\begin{array}{l}7.39 \pm \\
1.57\end{array}$ & $\begin{array}{l}7.82 \pm \\
1.23\end{array}$ & 0.02 & $\begin{array}{l}6.82 \pm \\
1.64\end{array}$ & $\begin{array}{l}8.26 \pm \\
1.29\end{array}$ & $0.001>$ & $\begin{array}{l}7.71 \pm \\
1.74\end{array}$ & $\begin{array}{l}7.13 \pm \\
1.30\end{array}$ & 0.05 & $\begin{array}{l}7.78 \pm \\
1.50\end{array}$ & $\begin{array}{l}6.96 \pm \\
1.37\end{array}$ & $0.001>$ \\
\hline $\begin{array}{l}\text { Vitamin } \\
\text { A(mcg/1000 } \\
\text { Kcal) }\end{array}$ & $\begin{array}{l}231.92 \\
\pm 449.62\end{array}$ & $\begin{array}{l}219.36 \\
\pm 115.93\end{array}$ & 1.00 & $\begin{array}{l}259.11 \\
\pm 142.31\end{array}$ & $\begin{array}{l}181.71 \\
\pm 90.12\end{array}$ & 0.01 & $\begin{array}{l}214.49 \\
\pm 129.77\end{array}$ & $\begin{array}{l}218.56 \\
\pm 116.54\end{array}$ & 1.00 & $\begin{array}{l}232.61 \\
\pm 127.26\end{array}$ & $\begin{array}{l}194.14 \\
\pm 3.79\end{array}$ & 0.77 \\
\hline $\begin{array}{l}\text { Vitamin } \\
\mathrm{C}(\mathrm{mg} / 1000 \mathrm{Kcal})\end{array}$ & $\begin{array}{l}33.64 \pm \\
22.90\end{array}$ & $\begin{array}{l}35.59 \pm \\
19.30\end{array}$ & 1.00 & $\begin{array}{l}42.23 \pm \\
25.79\end{array}$ & $\begin{array}{l}29.00 \pm \\
14.77\end{array}$ & $0.001>$ & $\begin{array}{l}31.73 \pm \\
24.42\end{array}$ & $\begin{array}{l}37.98 \pm \\
17.86\end{array}$ & 0.02 & $\begin{array}{l}35.75 \pm \\
21.35\end{array}$ & $\begin{array}{l}37.12 \pm \\
21.89\end{array}$ & 1.00 \\
\hline $\begin{array}{l}\text { Folate(mcg/1000 } \\
\text { Kcal) }\end{array}$ & $\begin{array}{l}233.89 \\
\pm 51.36\end{array}$ & $\begin{array}{l}227.86 \\
\pm 45.22\end{array}$ & 1.00 & $\begin{array}{l}221.2 \pm \\
58.22\end{array}$ & $\begin{array}{l}239.02 \\
\pm 47.39\end{array}$ & $0.01>$ & $\begin{array}{l}243.80 \\
\pm 55.62\end{array}$ & $\begin{array}{l}214.34 \\
\pm 41.4\end{array}$ & $0.001>$ & $\begin{array}{l}239.17 \\
\pm 53.07\end{array}$ & $\begin{array}{l}213.15 \\
\pm 44.78\end{array}$ & $0.001>$ \\
\hline $\begin{array}{l}\text { Vitamin } \\
\text { B6(mg/1000 } \\
\text { Kcal) }\end{array}$ & $\begin{array}{l}0.70 \pm \\
0.13\end{array}$ & $\begin{array}{l}0.73 \pm \\
0.12\end{array}$ & 0.15 & $\begin{array}{l}0.71 \pm \\
0.15\end{array}$ & $\begin{array}{l}0.70 \pm \\
0.10\end{array}$ & 1.00 & $\begin{array}{l}0.69 \pm \\
0.82\end{array}$ & $\begin{array}{l}0.73 \pm \\
0.12\end{array}$ & 0.04 & $\begin{array}{l}0.72 \pm \\
0.12\end{array}$ & $\begin{array}{l}0.69 \pm \\
0.12\end{array}$ & 0.08 \\
\hline $\begin{array}{l}\text { Vitamin } \\
\text { B12(mcg/1000 } \\
\text { Kcal) }\end{array}$ & $\begin{array}{l}1.78 \pm \\
4.55\end{array}$ & $\begin{array}{l}1.52 \pm \\
0.77\end{array}$ & 1.00 & $\begin{array}{l}1.73 \pm \\
0.88\end{array}$ & $\begin{array}{l}1.24 \pm \\
0.60\end{array}$ & 0.24 & $\begin{array}{l}1.43 \pm \\
0.82\end{array}$ & $\begin{array}{l}1.59 \pm \\
0.77\end{array}$ & 1.00 & $\begin{array}{l}1.53 \pm \\
0.85\end{array}$ & $\begin{array}{l}1.40 \pm \\
0.66\end{array}$ & 1.00 \\
\hline
\end{tabular}

Values are means \pm SD

1 obtained from one-way ANOVA

Association between food groups and depression, poor quality of life

Multi-variable adjusted odds ratios (ORs) for depression and poor QoL categories across quartiles of food intake are represented in Table 3. Adolescent girls in the fourth quartile of whole grain intake compared with the subjects in the first quartile had a $40 \%$ lower probability of having depressive symptoms (OR: 0.60 ; $95 \% \mathrm{Cl}: 0.37-0.98, \mathrm{p}=0.04)$. This association remained significant after adjustment for age, energy intake, BMI percentile, and physical activity $(\mathrm{OR}: 0.60 ; 95 \%$ $\mathrm{Cl}: 0.36-0.99, \mathrm{p}=0.04)$. There was a significant reduction trend in the odds of poor QoL across increasing quartile intake of whole grain in the crude (OR: 0.87 ; $95 \% \mathrm{Cl}: 0.76-0.99, \mathrm{p}$-trend $=0.04)$. This trend was not significant after adjustment for age, energy intake, BMI percentile, and physical activity $($ OR: $0.88 ; 95 \%$ Cl: $0.76-1.00, p$-trend $=0.06)$. Intake of refined grain was not associated with odds of depression and poor QoL $(\mathrm{OR}: 1.01 ; 95 \% \mathrm{Cl}$ : 0.66-1.52, P = 0.95). Subjects in the highest quartile of carbonated drink intake had higher odds of poor QoL compared with the subjects in the first quartile (OR: 1.33; $95 \% \mathrm{Cl}$ : $1.00-2.32, \mathrm{P}=0.04)$. This association remained significant after adjustment for age, energy intake, BMI percentile, and physical activity (OR: $1.69 ; 95 \% \mathrm{Cl}$ : $1.08-2.63, \mathrm{P}=0.02)$. However, intake of carbonated drink was not significantly associated with odds of depression (OR: 0.92; 95\% Cl: $0.57-1.47, \mathrm{p}=0.74)$. Fast food intake increased odds of poor QoL. The odds of poor QoL increased with higher intake of fast food (OR: 1.14; 95\% Cl: 1.00-1.37, P-trend = 0.04); after adjustment for potential confounders, this association remained significant (OR: 1.20; 95\% Cl: 1.04-1.38, P-trend =0.02). 
Table 3

Multivariable-adjusted odds ratio of the associations between depression, poor quality of life and food groups.

\begin{tabular}{|c|c|c|c|c|c|c|}
\hline \multirow{2}{*}{$\begin{array}{l}\text { Variable } \\
\text { Depression }\end{array}$} & \multicolumn{4}{|c|}{ Quartiles of whole grain intake } & \multirow[t]{2}{*}{ P value ${ }^{1}$} & \multirow[t]{2}{*}{$\mathrm{P}$ trend } \\
\hline & Q1 & Q2 & Q3 & Q4 & & \\
\hline Crude & 1.00 & $0.74\left(0.47 \_1.18\right)$ & $0.81\left(0.50 \_1.29\right)$ & 0.60 (0.37_0.98) & 0.04 & 0.06 \\
\hline Model1 & 1.00 & $0.75\left(0.47 \_1.19\right)$ & $0.81\left(0.51 \_1.30\right)$ & $0.61\left(0.37 \_1.00\right)$ & 0.05 & 0.08 \\
\hline Model2 & 1.00 & $0.75\left(0.47 \_1.18\right)$ & $0.82\left(0.51 \_1.32\right)$ & $0.61\left(0.37 \_1.01\right)$ & 0.05 & 0.09 \\
\hline Model3 & 1.00 & $0.74\left(0.47 \_1.18\right)$ & $0.80\left(0.50 \_1.29\right)$ & $0.60\left(0.36 \_0.99\right)$ & 0.04 & 0.07 \\
\hline \multicolumn{7}{|c|}{ Poor Quality of life } \\
\hline Crude & 1.00 & $0.89\left(0.59 \_1.34\right)$ & $0.71\left(0.46 \_1.09\right)$ & $0.67\left(0.47 \_1.03\right)$ & 0.07 & 0.04 \\
\hline Model1 & 1.00 & $0.87\left(0.58 \_1.32\right)$ & $0.70\left(0.46 \_1.07\right)$ & $0.66\left(0.43 \_1.01\right)$ & 0.05 & 0.03 \\
\hline Model2 & 1.00 & $0.87\left(0.58 \_1.32\right)$ & $0.71\left(0.46 \_1.09\right)$ & $0.66\left(0.43 \_1.02\right)$ & 0.06 & 0.03 \\
\hline \multirow[t]{2}{*}{ Model3 } & 1.00 & $0.88\left(0.58 \_1.33\right)$ & $0.73\left(0.48 \_1.12\right)$ & $0.69\left(0.45 \_1.07\right)$ & 0.10 & 0.06 \\
\hline & \multicolumn{6}{|c|}{ Quartiles of refined grain intake } \\
\hline Depression & Q1 & Q2 & Q3 & Q4 & & \\
\hline Crude & 1.00 & $0.69\left(0.43 \_1.13\right)$ & $0.81\left(0.50 \_1.30\right)$ & $1.02\left(0.65 \_1.62\right)$ & 0.48 & 0.76 \\
\hline Model1 & 1.00 & $0.72\left(0.44 \_1.18\right)$ & $0.88\left(0.54 \_1.46\right)$ & 1.21 (0.70_2.07) & 0.48 & 0.43 \\
\hline Model2 & 1.00 & $0.72\left(0.44 \_1.17\right)$ & $0.87\left(0.53 \_1.43\right)$ & 1.20 (0.70_2.05) & 0.50 & 0.46 \\
\hline Model3 & 1.00 & $0.71\left(0.44 \_1.17\right)$ & $0.87\left(0.53 \_1.43\right)$ & $1.21\left(0.70 \_2.08\right)$ & 0.47 & 0.44 \\
\hline \multicolumn{7}{|c|}{ Poor Quality of life } \\
\hline Crude & 1.00 & $0.73\left(0.48 \_1.01\right)$ & $0.86\left(0.57 \_1.30\right)$ & $1.01\left(0.66 \_1.52\right)$ & 0.95 & 0.76 \\
\hline Model1 & 1.00 & $0.75\left(0.49 \_1.14\right)$ & $0.91\left(0.59 \_1.40\right)$ & $1.14\left(0.70 \_1.85\right)$ & 0.57 & 0.48 \\
\hline Model2 & 1.00 & $0.74\left(0.48 \_1.12\right)$ & $0.89\left(0.57 \_1.37\right)$ & $1.12\left(0.69 \_1.82\right)$ & 0.62 & 0.53 \\
\hline Model3 & 1.00 & $0.74\left(0.49 \_1.13\right)$ & 0.89 (0.57_1.38) & $1.10\left(0.67 \_1.78\right)$ & 0.62 & 0.60 \\
\hline \multicolumn{7}{|c|}{ Quartiles of carbonated drink intake } \\
\hline Depression & Q1 & Q2 & Q3 & Q4 & & \\
\hline Crude & 1.00 & $0.92\left(0.57 \_1.47\right)$ & $0.86\left(0.54 \_1.38\right)$ & $0.92\left(0.57 \_1.47\right)$ & 0.74 & 0.85 \\
\hline Model1 & 1.00 & $0.78\left(0.48 \_1.26\right)$ & $0.88\left(0.55 \_1.41\right)$ & $0.98\left(0.60 \_1.60\right)$ & 0.94 & 0.96 \\
\hline Model2 & 1.00 & $0.79\left(0.49 \_1.27\right)$ & $0.88\left(0.55 \_1.42\right)$ & $0.98\left(0.60 \_1.60\right)$ & 0.94 & 0.96 \\
\hline Model3 & 1.00 & $0.78\left(0.48 \_1.26\right)$ & $0.87\left(0.54 \_1.40\right)$ & $0.97\left(0.59 \_1.59\right)$ & 0.91 & 0.99 \\
\hline \multicolumn{7}{|c|}{ Poor Quality of life } \\
\hline Crude & 1.00 & $0.87\left(0.57 \_1.31\right)$ & $1.03\left(0.68 \_1.56\right)$ & 1.33 (1.00_2.32) & 0.04 & 0.03 \\
\hline Model1 & 1.00 & $0.86\left(0.57 \_1.31\right)$ & $1.04\left(0.69 \_1.58\right)$ & $1.62\left(1.04 \_2.52\right)$ & 0.03 & 0.02 \\
\hline Model2 & 1.00 & $0.87\left(0.57 \_1.32\right)$ & 1.05 (0.69_1.59) & $1.63\left(1.05 \_2.53\right)$ & 0.02 & 0.02 \\
\hline Model3 & 1.00 & 0.88 (0.58_1.34) & $0.69\left(0.72 \_1.67\right)$ & 1.69 (1.08_2.63) & 0.02 & 0.01 \\
\hline \multicolumn{7}{|c|}{ Quartiles of fast food intake } \\
\hline Depression & Q1 & Q2 & Q3 & Q4 & & \\
\hline Crude & 1.00 & $1.16\left(0.71 \_1.89\right)$ & $1.37\left(0.85 \_2.20\right)$ & $1.20\left(0.74 \_1.98\right)$ & 0.46 & 0.36 \\
\hline Model1 & 1.00 & $1.21\left(0.74 \_2.98\right)$ & $1.46\left(0.89 \_2.39\right)$ & 1.35 (0.79_2.28) & 0.26 & 0.19 \\
\hline Model2 & 1.00 & $1.23\left(0.75 \_2.02\right)$ & 1.49 (0.91_2.43) & 1.37 (0.81_2.32) & 0.23 & 0.17 \\
\hline
\end{tabular}

${ }^{1}$ Fourth quartile compared to first quartile

Model 1: Adjusted for age and energy intake.

Model 2: additionally, adjusted for BMI percentile.

Model 3: additionally, adjusted for physical activity. 


\begin{tabular}{|c|c|c|c|c|c|c|}
\hline \multirow{2}{*}{$\begin{array}{l}\text { Variable } \\
\text { Model3 }\end{array}$} & \multicolumn{4}{|c|}{ Quartiles of whole grain intake } & \multirow{2}{*}{$\begin{array}{l}\text { P value }{ }^{1} \\
0.24\end{array}$} & \multirow{2}{*}{$\begin{array}{l}\mathrm{P} \text { trend } \\
0.18\end{array}$} \\
\hline & 1.00 & $1.23\left(0.75 \_2.01\right)$ & 1.47 (0.90_2.42) & $1.36\left(0.80 \_2.31\right)$ & & \\
\hline \multicolumn{7}{|c|}{ Poor Quality of life } \\
\hline Crude & 1.00 & $0.85\left(0.56 \_1.29\right)$ & 1.42 (0.94_2.15) & 1.33 (0.88_2.01) & 0.17 & 0.04 \\
\hline Model1 & 1.00 & 0.88 (0.57_1.33) & 1.51 (0.98_2.32) & 1.46 (0.93_2.22) & 0.09 & 0.01 \\
\hline Model2 & 1.00 & 0.89 (0.59_1.37) & 1.55 (1.01_2.38) & $1.49\left(0.95 \_2.34\right)$ & 0.07 & 0.01 \\
\hline Model3 & 1.00 & $0.90\left(0.59 \_1.38\right)$ & 1.59 (1.03_2.45) & 1.51 (0.96_2.38) & 0.07 & 0.01 \\
\hline \multicolumn{7}{|c|}{${ }^{1}$ Fourth quartile compared to first quartile } \\
\hline \multicolumn{7}{|c|}{ Model 1: Adjusted for age and energy intake. } \\
\hline \multicolumn{7}{|c|}{ Model 2: additionally, adjusted for BMI percentile. } \\
\hline \multicolumn{7}{|c|}{ Model 3: additionally, adjusted for physical activity. } \\
\hline
\end{tabular}

The correlations between intake of food groups with depression and QoL score are shown in Table 4. There was a positive association between intake of fast food and depression score in the crude model $(\beta=0.09$; P-value $=0.01)$. Likewise, this association remained significant after adjustment for age, energy intake, BMI percentile, and physical activity $(\beta=0.01 ; P$-value $<0.01)$. Intake of fast food was inversely associated with QoL score in the crude $(\beta=-0.081 ; P$-value $=$ 0.02 ) and adjusted models $(\beta=-0.090 ; P$-value $=0.02)$. Carbonated drink, refined grain and whole grain intake were not significantly associated with depression and QoL score.

Table 4

The association between Score of depression and quality of life with food

\begin{tabular}{|c|c|c|c|c|}
\hline \multirow[b]{2}{*}{ Food intake } & \multicolumn{2}{|c|}{ Score of Depression } & \multicolumn{2}{|c|}{ Score of Quality of life } \\
\hline & प & $P$ value & प & P value ${ }^{1}$ \\
\hline \multicolumn{5}{|c|}{ Intake of whole grain } \\
\hline Crude & -0.021 & 0.57 & 0.022 & 0.55 \\
\hline Model1 & -0.021 & 0.57 & 0.025 & 0.51 \\
\hline Model2 & -0.021 & 0.57 & 0.025 & 0.51 \\
\hline Model3 & -0.022 & 0.56 & 0.019 & 0.61 \\
\hline \multicolumn{5}{|c|}{ Intake of refined grain } \\
\hline Crude & 0.003 & 0.93 & 0.004 & 0.90 \\
\hline Model1 & 0.012 & 0.78 & 0.004 & 0.92 \\
\hline Model2 & 0.011 & 0.79 & 0.005 & 0.91 \\
\hline Model3 & 0.013 & 0.73 & 0.012 & 0.79 \\
\hline \multicolumn{5}{|c|}{ Intake of carbonated drink } \\
\hline Crude & 0.021 & 0.56 & -0.063 & 0.08 \\
\hline Model1 & 0.022 & 0.57 & -0.063 & 0.10 \\
\hline Model2 & 0.021 & 0.58 & -0.063 & 0.10 \\
\hline Model3 & 0.020 & 0.59 & -0.065 & 0.09 \\
\hline \multicolumn{5}{|c|}{ Intake of fast food } \\
\hline Crude & 0.090 & 0.015 & -0.081 & 0.02 \\
\hline Model1 & 0.101 & 0.009 & -0.088 & 0.02 \\
\hline Model2 & 0.102 & 0.009 & -0.089 & 0.02 \\
\hline Model3 & 0.102 & 0.009 & -0.090 & 0.02 \\
\hline \multicolumn{5}{|c|}{${ }^{1}$ Obtained from linear regression. } \\
\hline \multicolumn{5}{|c|}{ Model 1: Adjusted for age and energy intake. } \\
\hline \multicolumn{5}{|c|}{ Model 2: additionally, adjusted for BMI percentile. } \\
\hline Model 3: adc & ed for $\mathrm{ph}$ & cal activity. & & \\
\hline
\end{tabular}

Page $7 / 11$ 


\section{Discussion}

The high prevalence of depression and its outcomes like suicide ideation and suicide attempts in adolescents are important challenges for public health (1, 30 , 31). Modifying behaviors such as smoking, drinking, using drugs, physical activity, and diet may reduce the risk of depression and improve the QoL (32-34). Thus, evaluating the association of these modifiable factors with depression and QoL can be helpful for public health organizations, physicians, and patients for the management of depression and increasing QoL. To the best of our knowledge, the present study was the first cross-sectional study evaluating the association between specific food groups with depression and QoL among adolescent females.

We demonstrated that higher consumption of fast foods is positively associated with depression score. In addition, although based on the logistic regression analysis, a higher intake of whole grain is associated with lower odds of depression (at a slightly significant level with $\mathrm{P}=0.04$ ), according to linear regression analysis there was no association between whole grain intake and depression. Unlike linear regression analysis, in logistic regression analysis, individuals are categorized based their intakes and the linear relationship is not examined. Therefore, this finding must be interpreted with caution. However, we found no significant relationship between intake of refined grain and carbonated drink with risk of depression. There is no cross-sectional study examining the association between depression with the above-mentioned food groups among adolescents. A cross-sectional study that was conducted in adults showed an inverse relationship between frequency of healthy food groups such as fruits and vegetables consumption with odds of depression (35). This study used logistic regression analysis, but not linear regression analysis. Moreover, a study found that subjects without depression consumed more legumes, fruits, and vegetables compared to patients with depression (36). In addition, it has been shown that intake of non-refined grain is associated with lower odds of depression/anxiety disorders (37); but, high intake of refined grains was associated with a higher risk of depression (38). Some inconsistencies between the results of mentioned studies and our findings may be due to the differences in design, statistical analysis, methodology of the studies as well as age of participants. Our findings showed that subjects in the highest quartile of fast food and refined grain intake had higher energy intake which can induce obesity. Studies by Biobaku et al. (39), Guallar-Castillón et al. (40), Siracusa et al. (41) and Lang et al. (42) confirmed that fast foods and refined grains as the important components of unhealthy dietary patterns can induce obesity, oxidative stress and inflammation. Several investigations revealed the association between obesity, inflammation and oxidative stress with depression (8-10, 43). In addition, O'neil et al. (44) and Adjibade et al. (45) showed adherence to the healthy dietary patterns containing fruits, vegetables, and whole grains can reduce risk of depression.

On the other hand, the present study found that fast food consumption is inversely associated with the QoL score. Fast food is a component of unhealthy dietary patterns that can induce obesity, inflammation as well as oxidative stress (39-41), and subsequently increase the risk of depression (8-10). As mentioned, depression has a negative effect on QoL. Moreover, the logistic regression analysis showed higher carbonated drink consumption was associated with lower odds of poor QoL. However, linear model analysis demonstrated that there is no relationship between carbonated drink consumption and QoL score. As mentioned, unlike linear regression analysis, in the logistic regression analysis, subjects are categorized based their intakes and it cannot demonstrate the linear association. The results should be interpreted with caution. In addition, there was no significant association between intake of whole grain and refined grain with QoL score. There is no similar study in this field. A systematic review that conducted by Govindaraju et al. (46) confirmed that adherence to the healthy dietary patterns containing higher amounts of whole grains, fruits and vegetables as well as lower amounts of carbonated drinks and refined foods and fast food is directly associated with QoL score.

Some important strengths of our study are as follows: 1) The first study evaluating the association between specific food groups with depression among a relatively large sample of adolescents; 2) The first investigation examining the relationship between specific food groups with QoL; 3) High quality of data collection; and 4) Modulating the effects of various important confounding factors. However, the present study has some limitations. Due to design of the study, we cannot show the causality. Furthermore, this study was conducted only among adolescent females. Finally, low response and misclassification are common in cross-sectional studies, which can increase the probability of recall bias.

\section{Conclusions}

We demonstrated an inverse association between fast food intake with depression and QOL. However, there is no significant relationship between intake of refined grain and carbonated drink with depression. Furthermore, we found no association between intake of whole grain and refined grain with QoL. Due to inconsistent findings based on utilizing different analyses, the associations between intake of whole grain with depression as well as consumption of carbonated drink with QoL remained unclear. To better conclude, further studies evaluating the association of various food groups with depression and QoL.

\section{Abbreviations}

BDI

Beck depression inventory

BMI

body mass index

FFQ

food frequency questionnaire

MAQ

modifiable activity questionnaire

MUFA

monounsaturated fatty acids

OR

odds ratio 
PUFA

polyunsaturated fatty acids

QoL

quality of life

SD

standard deviation

SFA

saturated fatty acid

WC

waist circumference.

\section{Declarations}

\section{Ethics approval and consent to participate}

The ethical committee of Mashhad University of Medical Sciences in Mashhad approved the study (code number: 931188). All methods were performed in accordance with relevant guidelines. The written informed consent form was signed by all participants and their parents before the data collection.

\section{Consent for publication}

Not applicable.

\section{Availability of data and materials}

The data are not publicly available due to their containing information that could compromise the privacy of research participants. The data and materials of the current study is available from the corresponding author, Sayyed Saeid Khayyatzadeh, on reasonable request.

\section{Competing interests}

There is no conflict of interest.

\section{Funding}

The present study was supported by a grant provided by Mashhad University of Medical Sciences (MUMS).

\section{Authors' contributions}

MM conceived the idea. ZD and SK prepared the proposal. SK, MG obtained ethical approvals, applied for funding, provided data collection. ZD, AS wrote the manuscript. KL revised the manuscript. Authors read and approved the final manuscript.

Acknowledgements

The authors would like to thank all participants without whom this study was

impossible.

\section{References}

1. Collins PY, Patel V, Joestl SS, March D, Insel TR, Daar AS, et al. Grand challenges in global mental health. Nature. 2011;475(7354):27-30.

2. Murray CJ, Lopez AD. Evidence-based health policy-lessons from the Global Burden of Disease Study. Science. 1996;274(5288):740-3.

3. Prince M, Patel V, Saxena S, Maj M, Maselko J, Phillips MR, et al. No health without mental health. The lancet. 2007;370(9590):859 - 77.

4. Lépine J-P, Briley M. The increasing burden of depression. Neuropsychiatric disease and treatment. 2011;7(Suppl 1):3.

5. Belfer ML. Child and adolescent mental disorders: the magnitude of the problem across the globe. Journal of child psychology and psychiatry. 2008;49(3):226 - 36.

6. Cyranowski JM, Frank E, Young E, Shear MK. Adolescent onset of the gender difference in lifetime rates of major depression: a theoretical model. Archives of general psychiatry. 2000;57(1):21 - 7 .

7. Bijl RV, De Graaf R, Ravelli A, Smit F, Vollebergh WA. Gender and age-specific first incidence of DSM-III-R psychiatric disorders in the general population. Social psychiatry and psychiatric epidemiology. 2002;37(8):372-9.

8. Shafiee M, Ahmadnezhad M, Tayefi M, Arekhi S, Vatanparast H, Esmaeili H, et al. Depression and anxiety symptoms are associated with prooxidantantioxidant balance: A population-based study. Journal of affective disorders. 2018;238:491-8. 
9. Shafiee M, Tayefi M, Hassanian SM, Ghaneifar Z, Parizadeh MR, Avan A, et al. Depression and anxiety symptoms are associated with white blood cell count and red cell distribution width: a sex-stratified analysis in a population-based study. Psychoneuroendocrinology. 2017;84:101-8.

10. Tayefi M, Shafiee M, Kazemi-Bajestani SMR, Esmaeili H, Darroudi S, Khakpouri S, et al. Depression and anxiety both associate with serum level of hs-CRP: a gender-stratified analysis in a population-based study. Psychoneuroendocrinology. 2017;81:63-9.

11. Sajjadi H, Kamal SHM, Rafiey H, Vameghi M, Forouzan AS, Rezaei M. A systematic review of the prevalence and risk factors of depression among Iranian adolescents. Global journal of health science. 2013;5(3):16.

12. Teles F, Amorim de Albuquerque AL, Freitas Guedes Lins IK, Carvalho Medrado P, Falcão Pedrosa Costa A. Quality of life and depression in haemodialysis patients. Psychology, health \& medicine. 2018;23(9):1069-78.

13. Gao K, Su M, Sweet J, Calabrese JR. Correlation between depression/anxiety symptom severity and quality of life in patients with major depressive disorder or bipolar disorder. Journal of affective disorders. 2019;244:9-15.

14. Sivertsen H, Bjørkløf GH, Engedal K, Selbæk G, Helvik A-S. Depression and quality of life in older persons: a review. Dementia and geriatric cognitive disorders. 2015;40(5-6):311-39.

15. Group: TW. The World Health Organization Quality of Life Assessment (WHOQOL): position paper from the World Health Organization. Soc Sci Med. 1995;41:1409.

16. Weng T-T, Hao J-H, Qian Q-W, Cao H, Fu J-L, Sun Y, et al. Is there any relationship between dietary patterns and depression and anxiety in Chinese adolescents? Public health nutrition. 2012;15(4):673 - 82.

17. Oddy WH, Allen KL, Trapp GS, Ambrosini GL, Black LJ, Huang R-C, et al. Dietary patterns, body mass index and inflammation: pathways to depression and mental health problems in adolescents. Brain, behavior, and immunity. 2018;69:428 - 39.

18. Park S-J, Kim M-S, Lee H-J. The association between dietary pattern and depression in middle-aged Korean adults. Nutrition research and practice. 2019;13(4):316.

19. Li Z, Li B, Song X, Zhang D. Dietary zinc and iron intake and risk of depression: A meta-analysis. Psychiatry research. 2017;251:41 - 7.

20. Li Z, Wang W, Xin X, Song X, Zhang D. Association of total zinc, iron, copper and selenium intakes with depression in the US adults. Journal of affective disorders. 2018;228:68-74.

21. Liu X, Yan Y, Li F, Zhang D. Fruit and vegetable consumption and the risk of depression: a meta-analysis. Nutrition. 2016;32(3):296-302.

22. Anjom-Shoae J, Sadeghi O, Keshteli AH, Afshar H, Esmaillzadeh A, Adibi P. Legume and nut consumption in relation to depression, anxiety and psychological distress in Iranian adults. European journal of nutrition. 2020:1-11.

23. Carson TL, Hidalgo B, Ard JD, Affuso O. Dietary interventions and quality of life: a systematic review of the literature. Journal of nutrition education and behavior. 2014;46(2):90-101.

24. Asghari G, Rezazadeh A, Hosseini-Esfahani F, Mehrabi Y, Mirmiran P, Azizi F. Reliability, comparative validity and stability of dietary patterns derived from an FFQ in the Tehran Lipid and Glucose Study. British journal of nutrition. 2012;108(6):1109-17.

25. Pehrsson P, Haytowitz D, Holden J, Perry C, Beckler D. USDA's national food and nutrient analysis program: food sampling. Journal of Food Composition and Analysis. 2000;13(4):379-89.

26. Ghassemzadeh H, Mojtabai R, Karamghadiri N, Ebrahimkhani N. Psychometric properties of a Persian-language version of the Beck Depression InventorySecond edition: BDI-II-PERSIAN. Depression and anxiety. 2005;21(4):185 - 92.

27. Scogin F, Beutler L, Corbishley A, Hamblin D. Reliability and validity of the short form Beck Depression Inventory with older adults. Journal of Clinical Psychology. 1988;44(6):853-7.

28. Cheak-Zamora NC, Wyrwich KW, McBride TD. Reliability and validity of the SF-12v2 in the medical expenditure panel survey. Quality of Life Research. 2009;18(6):727 - 35.

29. Montazeri A, Vahdaninia M, Mousavi SJ, Asadi-Lari M, Omidvari S, Tavousi M. The 12-item medical outcomes study short form health survey version 2.0 (SF-12v2): a population-based validation study from Tehran, Iran. Health and quality of life outcomes. 2011;9(1):1-8.

30. Kessler RC, Angermeyer M, Anthony JC, De Graaf R, Demyttenaere K, Gasquet I, et al. Lifetime prevalence and age-of-onset distributions of mental disorders in the World Health Organization's World Mental Health Survey Initiative. World psychiatry. 2007;6(3):168.

31. Turecki G, Brent DA. Suicide and suicidal behaviour. The Lancet. 2016;387(10024):1227-39.

32. Michael SL, Lowry R, Merlo C, Cooper AC, Hyde ET, McKeon R. Physical activity, sedentary, and dietary behaviors associated with indicators of mental health and suicide risk. Preventive medicine reports. 2020;19:101153.

33. Dowdy E, Furlong MJ, Sharkey JD. Using surveillance of mental health to increase understanding of youth involvement in high-risk behaviors: A valueadded analysis. Journal of Emotional and Behavioral Disorders. 2013;21(1):33-44.

34. Lowry R, Crosby AE, Brener ND, Kann L. Suicidal thoughts and attempts among US high school students: trends and associated health-risk behaviors, 1991-2011. Journal of Adolescent Health. 2014;54(1):100-8.

35. Bishwajit G, O'Leary DP, Ghosh S, Sanni Y, Shangfeng T, Zhanchun F. Association between depression and fruit and vegetable consumption among adults in South Asia. BMC psychiatry. 2017;17(1):1-9.

36. Grases G, Colom M, Sanchis P, Grases F. Possible relation between consumption of different food groups and depression. BMC psychology. 2019;7(1):16.

37. Gibson-Smith D, Bot M, Brouwer IA, Visser M, Giltay EJ, Penninx BW. Association of food groups with depression and anxiety disorders. European journal of nutrition. 2020;59(2):767- 78 . 
38. Moludi J, Moradinazar M, Hamzeh B, Najafi F, Soleimani D, Pasdar Y. Depression Relationship with Dietary Patterns and Dietary Inflammatory Index in Women: Result from Ravansar Cohort Study. Neuropsychiatric Disease and Treatment. 2020;16:1595.

39. Biobaku F, Ghanim H, Batra M, Dandona P. Macronutrient-mediated inflammation and oxidative stress: relevance to insulin resistance, obesity, and atherogenesis. The Journal of Clinical Endocrinology \& Metabolism. 2019;104(12):6118-28.

40. Guallar-Castillón P, Rodríguez-Artalejo F, Fornés NS, Banegas JR, Etxezarreta PA, Ardanaz E, et al. Intake of fried foods is associated with obesity in the cohort of Spanish adults from the European Prospective Investigation into Cancer and Nutrition. The American journal of clinical nutrition. 2007;86(1):198-205.

41. Siracusa F, Schaltenberg N, Villablanca EJ, Huber S, Gagliani N. Dietary habits and intestinal immunity: from food intake to CD4 + TH cells. Frontiers in immunology. 2019;9:3177.

42. Lang UE, Beglinger C, Schweinfurth N, Walter M, Borgwardt S. Nutritional aspects of depression. Cellular Physiology and Biochemistry. 2015;37(3):102943.

43. Anderson SE, Cohen P, Naumova EN, Jacques PF, Must A. Adolescent obesity and risk for subsequent major depressive disorder and anxiety disorder: prospective evidence. Psychosomatic medicine. 2007;69(8):740-7.

44. O'neil A, Quirk SE, Housden S, Brennan SL, Williams LJ, Pasco JA, et al. Relationship between diet and mental health in children and adolescents: a systematic review. American journal of public health. 2014;104(10):e31-e42.

45. Adjibade M, Assmann KE, Andreeva VA, Lemogne C, Hercberg S, Galan P, et al. Prospective association between adherence to the Mediterranean diet and risk of depressive symptoms in the French SU. VI. MAX cohort. European journal of nutrition. 2018;57(3):1225-35.

46. Govindaraju T, Sahle BW, McCaffrey TA, McNeil JJ, Owen AJ. Dietary patterns and quality of life in older adults: a systematic review. Nutrients. 2018;10(8):971. 